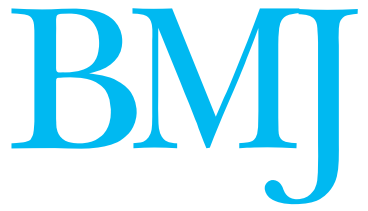

\title{
Managing minor head injury in children
}

\author{
Early discharge after computed tomography is safe and cost effective
} Research pp 465,
469
$\mathrm{I}$ n the United Kingdom some 500000 children attend accident and emergency departments after a head injury and about 50000 are admitted (nearly $10 \%$ of all hospital admissions). The death rate is 5.3 per 100000 children. ${ }^{1}$ Despite various guidelines on the management of head injury, clinical practice varies. Two papers in this week's $B M J$ shed new light on the management of mild head injury in children admitted to emergency departments. ${ }^{23}$ They compare immediate computed tomography for triage with inpatient observation in mild head injury-920 of the 2602 enrolled patients were aged 6-15. No child died or needed surgical intervention. Early discharge after immediate computed tomography was no worse in terms of recovery at three months, later complications, and patient satisfaction; it was also less costly than inpatient observation.

Here is a scenario familiar to emergency department doctors, paediatricians, and general practitioners. A child suffers apparently mild head trauma with brief loss of consciousness. When seen, he or she has normal neurological findings and a Glasgow coma scale score of 15 . The child makes an uninterrupted recovery but spends 24 hours in hospital, with worried parents at the bedside and nurses performing hourly neurological observations of variable quality. Fortunately a less common scenario is that, in the hour or so between the decision to admit the child and actual admission to the ward, he or she develops neurological signs from an unsuspected subdural haematoma and is left with permanent sequelae, despite transfer to a neurosurgical centre. How then, do we differentiate between children who need urgent imaging and referral to a neurosurgical service and those who need observation only?

A literature review designed to inform a clinical practice guideline for the American Academy of Pediatrics found 108 relevant studies before 1999, but it concluded that there was not sufficient scientific basis for evidence based recommendations on key management issues. ${ }^{4}$ A subsequent meta-analysis of studies investigating clinical variables that might predict serious intracranial injury in children showed, unsurprisingly, that predictors include reduced level of consciousness, focal neurological signs, and skull fracture. Neither headache (difficult or impossible to recognise in children who have not yet started to speak) nor vomiting increased the risk..$^{5}$

Skull radiography was used for many decades to identify children at greatest risk. However, although skull fractures increase the risk of intracranial injury fourfold, the presence of a fracture is of limited value as a diagnostic test-with a calculated sensitivity of $59 \%$ and specificity of $88 \% .^{5}$ A retrospective audit of two cohorts of children presenting to an emergency department in a UK paediatric teaching hospital (before and after restricting the use of skull $\mathrm{x}$ rays) concluded that abolishing the use of $\mathrm{x}$ rays, except in infants under 12 months, did not increase the admission rate or the rate of missed intracranial injury and reduced overall radiation dosage, despite doubling the proportion of children who receive a computed tomography scan from $1.0 \%$ to $2.1 \%{ }^{6}$ The authors' protocol permitted skull $\mathrm{x}$ rays for the youngest infants if non-accidental injury was suspected or evidence of head trauma existed. ${ }^{7}$

In June 2003, the National Institute for Clinical Excellence (NICE) published clinical guidelines for managing head injury; these guidelines were mainly based on the Canadian computed tomography head rule and the New Orleans criteria. ${ }^{89}$ Both these sets of criteria are $100 \%$ sensitive for the need for neurosurgical intervention, and the Canadian rules have higher specificity for important clinical outcomes $(50.6 \% v$ $12.7 \%$ ) so are likely to result in lower imaging rates. ${ }^{10}$

These guidelines advocate a strategy based on early computed tomography scanning for patients deemed clinically to be at high risk and a major reduction in the use of skull $x$ rays. Audits in the UK, conducted since the guidelines were adopted, have shown an increase in the incidence of performing computed tomography head scans: from $1-2 \%$ to $7-9 \%$ of attendees of all ages in hospitals following NICE guidelines ${ }^{11} 12$ and from $14 \%$ to $20 \%$ at a hospital following the Canadian rules. ${ }^{13}$

The practicability of applying any of these guidelines will depend to some extent on the availability of staff and imaging facilities and the cash to pay for them in a clinical situation more commonly met out of hours. One question that arises is whether extra expenditure on scanning might be balanced by savings on hospital admission-unlikely in circumstances where even patients with a normal scan might still be admitted.

Clinicians may have little difficulty in predicting the response of their radiological colleagues to a request to image all patients presenting with minor head injury, however much they point out the savings of about $€ 200$ ( $£ 135$; \$256) per patient calculated by the Swedish group. ${ }^{3}$ To convince radiologists, further studies are needed to define a highest risk group who could be scanned on arrival then discharged (or x rayed if under 12 months) and a lowest risk group who could be sent 
home from an observation unit without being scanned. Two subjective issues would need to be dealt with by qualitative research: the degree to which patients are reassured by (unnecessary) imaging thereby preventing re-attendance, and the degree to which doctors' (possibly irrational) fears of medicolegal consequences of not imaging can be assuaged.

Harvey Marcovitch associate editor, BMJ

BMJ Editorial, Tavistock Square, London WC1H 9JR

(h.marcovitch@btinternet.com)

Competing interests: None declared.

Jennett B. Epidemiology of head injury. Arch Dis Child 1998;78:403-6.

2 af Geijerstam J-L, Oredsson S, Britton M, for the OCTOPUS Study Investigators. Medical outcome after immediate computed tomography or admission for observation in patients with mild head injury: randomised controlled trial. BMJ 2006 doi: 10.1136/bmj.38918 669317.4F.

3 Norlund A, Marké L-Å, af Geijerstam J-L, Oredsson S, Britton M, for the OCTOPUS. Cost comparison of immediate computed tomography or OCIOPU. Cost companson of immediate compun trial. BMJ 2006 doi: $10.1136 /$ bmj. $38918.659120 .4 \mathrm{~F}$.

4 Homer CJ, Kleinman L. Technical report: minor head injury in children. Homer CJ, Kleinman L.
5 Dunning J, Batchelor J, Stratford-Smith P, Teece S, Browne J, Sharpin C, et al. A meta-analysis of variables that predict significant intracranial et al. A meta-analysis of variables that predict significan
injury in minor head trauma. Arch Dis Child 2004;89:653-9.

injury in minor head trauma. Arch Dis Child 2004;89:653-9.
Reed MJ, Browning JG, Wilkinson AG, Beattie T. Can we abolish skull x rays for head injury? Arch Dis Child 2005;90:859-64.

Browning JG, Reed MJ, Wilkinson AG, Beattie T. Imaging infants with head injury: effect of a change in policy. Emerg Med J 2005;22:33-6.

8 National Institute for Clinical Excellence. Head injury. Triage, assessment, investigation and early management of head injury in infants, children and adults. Clinical Guideline 4. Developed by the National Collaborating Centre for Acute Care London: NTCE 2003. wwwnice or

Stiell IG, Well GA, Vandem St. al. The Canadian CT head rule for patients with minor head injury. Lancet 2001;357:1391-6.

10 Stiell IG, Clement CM, Rowe BH, Schull MJ, Brison R, Cass D, et al. Comparison of the Canadian CT head rule and the New Orleans criteria in patients with minor head injury. JAMA 2005;294:1511-8.

11 Shravat BP, Huseyin TS, Hynes KA. NICE guideline for the management of head injury: an audit demonstrating its impact on a district general hospital, with a cost analysis for England \& Wales. Emerg Med J 2006;23:109-13

12 Hassan Z, Smith M, Littlewood S, Bouamra O, Hughes D, Biggin C, et al. Head injuries: a study evaluating the impact of the NICE head injury Head inju

13 Sultan HY, Boyle A, Pereira M, Antoun N, Maimaris C. Application of the Canadian CT head rules in managing minor head injuries in a UK emergency department: implications for the implementation of the NICE guidelines. Emerg Med J 2004;21:420-5.

doi 10.1136/bmj.38954.516933.DE

\section{Growing pains}

\section{Parents and children need reassuring about this self limiting condition of unknown cause}

G rowing pains are typically non-articular, intermittent bilateral aches or pains in the legs that occur in the evening or at night in children aged 3-12 years. They are not associated with limping or limited mobility and do not involve the joints (all of which are recognised signs of pathology); no signs of local trauma or infection are seen. ${ }^{1}$ Physical examination and laboratory and $\mathrm{x}$ ray investigations are normal. The diagnosis of growing pains is one of exclusion. Reported prevalence ranges from 2.6\% to $49.4 \%$, which reflects the diverse criteria used to identify cases and the differing populations sampled.

What causes growing pains? The short answer is that we still do not know. Although the term has been used for more than 180 years it has proved to be a misnomer-growth spurts do not play a part in this condition. $^{2}$ Many aetiologies have been proposed but have not been supported by subsequent research. The idea that growing pains are caused by rheumatic fever was disproved in the 1930s. ${ }^{3}$ Subsequent causal theories include faulty posture and an association with restless leg syndrome, vascular perfusion disorder, fatigue, or emotional disturbance.

Only one small study has shown a relation between growing pains and posture, and it suggested a possible association with pronated foot. ${ }^{\mathrm{w1}}$ Restless leg syndrome in children is sometimes misdiagnosed as growing pains, but unlike growing pains this syndrome persists into adulthood. One small study found that children with growing pains were more likely than others to have a parent with a history of restless leg syndrome. ${ }^{\text {w2 }}$ A study comparing bone scans of children with growing pains with those of controls concluded that growing pains are not associated with changes in vascular perfusion. ${ }^{\mathrm{w} 3}$
One study found that children with growing pains had lower tibial bone strength than controls. ${ }^{\mathrm{w4}}$ This supports the hypothesis that growing pains are a symptom of "overuse syndrome of the lower extremities" with bone fatigue. However, growing pains are not associated with bone fracture. The study provided insufficient information to determine whether the children had increased pain after physical activity.

Another study found that children with "osteomuscular pains of unknown origin" (synonymous with growing pains) had markedly increased levels of lead in their scalp hair compared with children of similar age ${ }^{w 5}$ The diagnostic criteria for growing pains were ill defined, however, and the person who conducted the assay did not seem to be blinded to the children's diagnoses.

Because the pathogenesis of growing pains is unclear, psychological factors have been suggested to have a role, but no convincing evidence has been found. ${ }^{4}$ In an Australian longitudinal study of temperament, children with growing pains were more likely than controls to be rated by their parents as also having abnormal pains, negative moods, or behavioural problems, although the children's teachers rated them the same as the controls for these measures. ${ }^{5}$ In a study of school children, episodes of limb pain were reported to have similar trigger factors, associated symptoms, and relieving factors to episodes of headache in children with migraine. ${ }^{6}$ No differences in personality were noted between children with growing pains and controls. Another study found that children with growing pains had lower pain thresholds than children of similar age and 